\title{
RECENT ADVANCES IN GOLD-NHC COMPLEXES WITH BIOLOGICAL PROPERTIES
}

Mora, Malka; Gimeno Concepción, María; Visbal, Renso

\begin{abstract}
This tutorial review covers the recent advances made in the study of gold complexes containing $\mathrm{N}$-heterocyclic carbene ligands with biological properties. The great stability, ease of modulation of the electronic properties and excellent $\sigma$-donating capacity displayed by NHCs allow gold-NHC derivatives to reach high stability in biological media and relatively good internalization into cells and for that they have emerged as excellent potential chemotherapeutics. The new gold-NHC derivatives show superior anticancer activity compared to other standards such as Cisplatin or Auranofin. In addition, the application of gold-NHC complexes in the treatment of other human diseases as antibacterial, antioxidant and antiparasitic agents is reviewed for the first time
\end{abstract}

Keyword

Antioxidant, Carbene, Coordination compound, Gold, Heterocyclic compound, Methane 